Document downloaded from:

http://hdl.handle.net/10251/37458

This paper must be cited as:

Palanca Cámara, J.; Navarro Llácer, M.; Julian Inglada, VJ.; García-Fornes, A. (2012). Distributed goal-oriented computing. Journal of Systems and Software. 85(7):1540-1557. doi:10.1016/j.jss.2012.01.045.

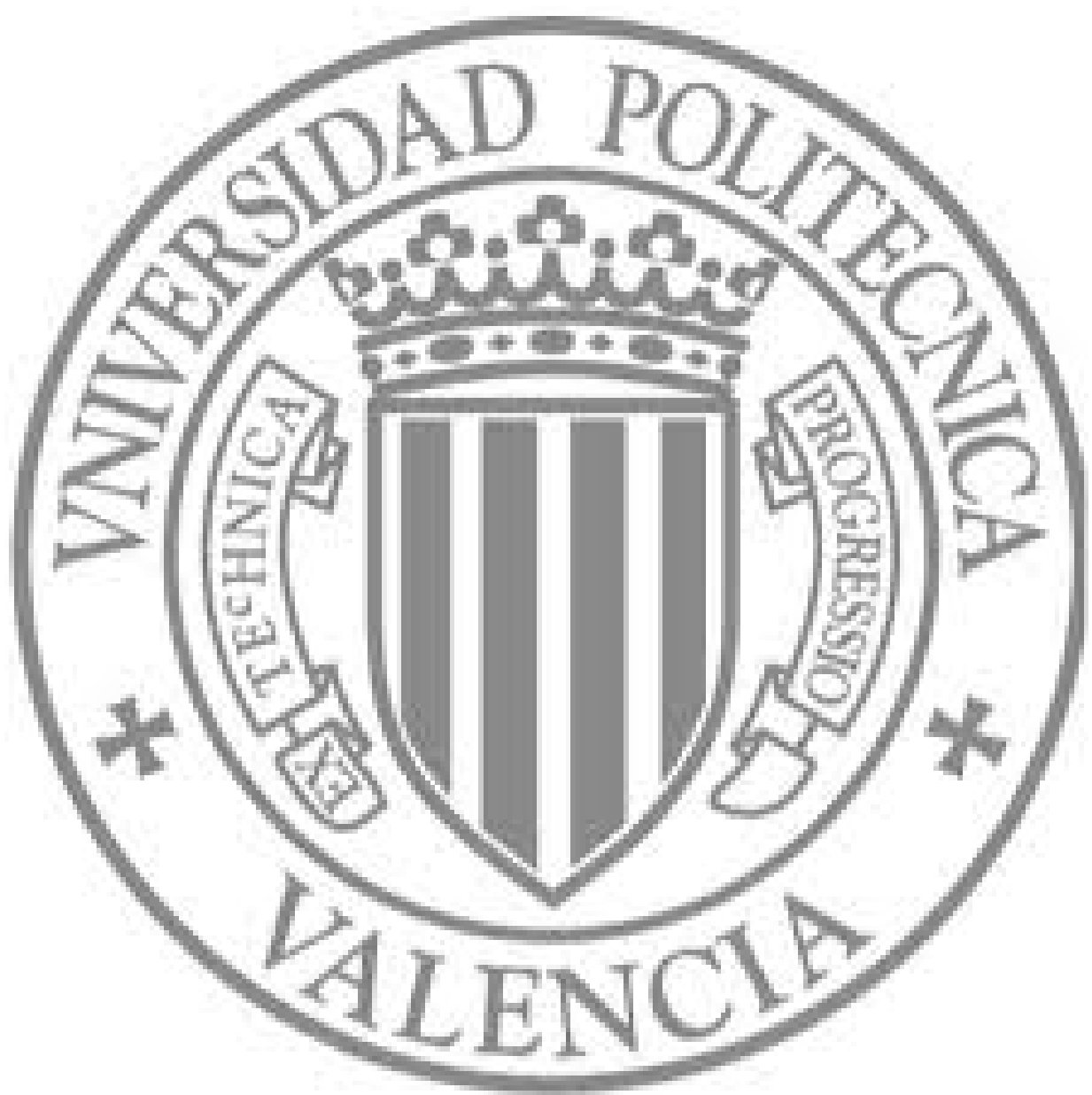

The final publication is available at

http://dx.doi.org/10.1016/j.jss.2012.01.045

Copyright Elsevier 


\title{
Distributed Goal-Oriented Computing
}

\author{
Javier Palanca, Martí Navarro, Vicente Julian, Ana García-Fornes \\ DSIC - Universitat Politècnica de València \\ Camino de Vera $s / n, 46022$ \\ Valencia, SPAIN
}

\begin{abstract}
For current computing frameworks, the ability to dynamically use the resources that are allocated in the network has become a key success factor. As long as the size of the network increases, it is more difficult to find how to solve the problems that the users are presenting. Users usually do know what they want to do, but they don't know how to do it. If the user knows its goals it could be easier to help him with a different approach. In this work we present a new computing paradigm based on goals. This paradigm is called Distributed Goal-Oriented Computing paradigm. To implement this paradigm an execution framework for a Goal-oriented Operating System has been designed. In this paradigm users express their goals and the OS is in charge of helping the achievement of these goals by means of a service-oriented approach.
\end{abstract}

\section{Introduction}

The amount of developed software and its complexity has currently increased so much that it has lead to discover that traditional paradigms of software development are not enough to create complex software. That is why there is a constant work on new paradigms, to improve the level of abstraction needed to develop increasingly complex applications. Among these paradigms, we can highlight the Service-Oriented Computing paradigm and Multi-Agent Systems.

Service-Oriented Computing (SOC) is a paradigm where the fundamental component for developing applications is the service. Using single services or service compositions it is possible to achieve solutions to problems in a decentralized manner with a high degree of adaptability. This paradigm, coupled with the cloud-computing one, is becoming very important at the present moment because both paradigms allow us to develop applications based on platformagnostic, distributed and low-cost computational elements. The use of SOC in multi-agent systems is endorsed by the proposal of achieving the agent goals by

Email addresses: jpalanca@dsic.upv.es (Javier Palanca), mnavarro@dsic.upv.es (Martí Navarro), vinglada@dsic.upv.es (Vicente Julian), agarcia@dsic.upv.es (Ana García-Fornes)

Preprint submitted to Journal of Systems and Software

August 15, 2011 
means of the invocation and composition of a set of services that are available within the multi-agent system.

Dickinson and Wooldridge discuss at [22] different ways to consider the relationship between multi-agent systems and service architectures. As it is summarized in that work, some authors propose that there is no conceptual distinction between agents and services: both are active building blocks in a loosely coupled architecture [3]. Another approach considers a bi-directional integration where agents and services interoperate by communicating one to each other [7]. Finally, a third approach considers that agents are who invoke services [20]. In this proposal, agents mediate between services and users.

Since agents are intelligent entities and have social capabilities, they fit properly in a service-based framework[15] where the goal-oriented computing approach is used. This approach is based on finding solutions to problems through composition and execution of various services offered by different agents.

This goal-oriented computing paradigm suggests that agents provide services in a ubiquitous environment and users only need to express their goals. Thereby users can reach a solution by finding a plan which achieves the selected goal with very limited and simplified user interaction.

This functionality should be provided to agents through a specific framework that supports service composition and their subsequent execution. Agents are providers and consumers of services in this framework, where agents use their social capabilities to find a way to fulfill their own goals. These capabilities should be provided to agents through a specific architecture that supports service composition and their subsequent execution. This framework is presented in this work as an execution module for a Goal-oriented Operating System. Current operating systems (OS) are based on abstractions that have not evolved too much since their first designs. However, the evolution of software engineering poses the possibility of addressing the OS design from other points of view. The Distributed Goal-Oriented Computing paradigm offers new ideas for the development of more intelligent and effective OS's, which would benefit the end-user due to the advantages of both technologies.

In this work the Distributed Goal-Oriented Computing paradigm is presented. It is also presented an execution module for a Goal-oriented Operating System which gives support to this paradigm following the requirements defined in this work. Some of this requirements comprise how to define the properties of a goal and the parameters that define how good is a plan. Some of the parameters that involve the creation and selection of a plan are time and trust.

This paper is structured as follows: Section 2 presents a related work about Operating System designs and trends. Section 3 presents the model and the architecture of the Distributed Goal-Oriented paradigm used in this work. In Section 4 we talk about the operating system deliberation engine and the various components that comprise this engine. Section 5 presents the execution module that interacts with the deliberation engine to develop the presented paradigm. This module is the Runtime Engine. Section 6 presents a series of experiments to show the functionality of this Operating System. Finally, Section 7 presents the conclusions of this work. 


\section{Related Work}

Operating Systems research is always trying to improve security, efficiency and reliability of Operating Systems. This is one of the great challenges of the current OS that remains to be overcome. Several studies have focused on improving certain OS aspects as data access or the input/output (I/O) abstractions, leading to propose new abstractions in this field (file, object, socket, ...) $[16,14,18]$. However, no significant progress has been made in implementing OS execution models.

Nowadays, any operating system has multiuser, interactive and multiprocessor skills due to the evolution of computers, which made indispensable that all the OS endure it. The interesting point is the vision of its purpose, due to the important differences that lie on an operating system's design depending on what it was created for. It depends on how the device is going to be used for or its specific functionality. The following is an updated classification that includes the different OS differentiated by their architecture or purpose. This is not an exclusive classification, since it represents the different approaches that can be taken during the design of an OS. Several options can be taken simultaneously. This makes possible the creation of, for example, a multiprocessor, extensible and general purpose operating system.

- Mainframe operating systems: These are systems oriented to large computers where the computing and the input/output (E/S) power are important.

- Server operating systems: they are oriented to bring services across the net as well as to process efficiently a large amount of requests per second.

- General purpose operating systems: they were created for mass consumption. Their only goal is to bring, with a simple and friendly interface, the most common tools for the daily use of a personal computer.

- Extensible operating systems: Extensible OS give support to dynamic loading of new features in the system as required for its purpose. These new modules are loaded to extend the OS according to the needs of each moment.

- Multiprocessor operating systems: A special OS is needed to handle and share the jobs in computers with more than one CPU.

- Parallel operating systems: These are an extension to multiprocessor systems where the need to run different applications on multiple processors extends to a computer network or cluster.

- Distributed operating systems: Nowadays, there is a trend towards distribution of the different services that an OS offers among a number of computers making use of the network. 
- Grid operating systems: These are an extension of distributed systems where there is access to geographically distributed resources by all network nodes in a heterogeneous grid.

- Real Time operating systems: These are OS for some very specific applications where not only the result of an operation is provided, but also the precise moment is important.

- Embedded operating systems: These systems run on control devices which are not generally though as real computers and which do not accept user-installed systems. Typical examples are microwave ovens, washing machines, televisions, cars, etc.

On this list of operating systems we can distinguish several groups that will outline the current trends in OS development. Thus, operating systems are characterized as service-oriented (such as servers), those aimed at enhancing performance or availability of the network (such as distributed systems, grid, parallel, etc.), those systems oriented to a particular purpose (such as real-time or embedded ones). Embedded systems become day to day more important due to the increasingly massive introduction of mobile devices. Finally, the everpresent general purpose operating systems, which are still very important given the high penetration of personal computers in homes.

One reason why no significant progress has been made in the OS execution model abstractions, such as the process, is that these abstractions are closely tied to current hardware. Processors are designed to work optimally with processes. Thereby, when adding improvements to the OS execution model, as well as defining new execution abstractions (as proposed in this paper) would be interesting to start thinking about adapting the hardware to such abstractions.

\subsection{Three Modern Operating Systems}

In this section three modern Operating Systems are presented and analyzed in order to study the new trends in OS design and implementation. The OS analyzed are Singularity (an experimental OS where have been tested new techniques like code verification, contracts or modern VM-based languages), MINIX 3 (an evolution of the classic MINIX OS where the focus is on miniaturization of the microkernel and embedded systems), and finally XtreemOS (a distributed OS based on organizations and built on top of Linux). Some ideas about these three OS are presented below.

\subsubsection{Singularity}

Singularity $[10,9]$ is an experimental OS developed by Microsoft Research in 2003. Its main objective is to achieve high reliability. For this reason they have started the development of the OS from scratch. It has been possible to experiment with new technologies and high-level languages to build the architecture of the OS, this way they've achieved a very robust and reliable system. Therefore, one of its most critical abstractions are Software-Isolated Processes 
(SIP), which represent Singularity processes. Any code running outside the kernel is running in a SIP. The SIP is a way of encapsulating software into separate and fault tolerant components.

\subsubsection{MINIX 3}

MINIX is one of the most popular microkernel OS still in development. Originally designed by Andrew S. Tanenbaum as a study Operating System for his students. Its design was a model for the construction of other Operating Systems, while it has continued its own evolution, reaching in 2006 the third version of the OS: MINIX 3[8].

The main objective of the third version of MINIX is reliability, devising for it a self-reparable system. They have followed the design philosophy of microkernel, leaving in protected-mode the minimal functionality and placing in user-mode all the remaining functionality. Thus, the user-mode failures are not critical for the system and also, due to a system called Reincarnation Server, failing processes are self-reparable and can be relaunched in the same state they have failed.

\subsubsection{XtreemOS}

$\mathrm{XtreemOS}[4,11]$ is a Grid Operating System. The development of this system is based on the Linux OS and its objectives are transparency and scalability. Transparency is offered to both the user and the application, since the great advantage of XtreemOS is still offering a Linux interface despite the availability of certain services and resources distributed on the network. Furthermore, this transparency allows heterogeneity among the classic applications of Linux and those found in the Grid.

XtreemOS uses virtual organizations (VO) to encapsulate the services and resources in the Grid. A VO administrator is responsible for its creation, management and completion, whether it is static and dynamic.

\subsection{Discussion}

The biggest innovation in the field of operating systems has probably been the introduction and expansion of the network. The leap from single centralized computing in a distributed computing in all computers across the net, which is called cloud, has emerged a complete redesign of the operating systems to adapt themselves to this new technology.

The functionality that is demanded today from an OS has changed from what was being demanded lately. Factors such as cross-platform, multi-processor support or concurrency ability do not pose a technological challenge today, as we discussed earlier, and are in the vast majority of new developments in operating systems. Key factors that make a difference in the new OS are related to the network (such as being distributed, single image, access to services, transparency ...) and those related to security and integrity of information. Two other important factors remain the efficiency of the system (as much as you increase the speed of the hardware it is still important that the OS interferes as little 
as possible in the response time of applications), and one factor which becomes more important every day because the increasing complexity of applications: reliability.

Our proposal is to focus on major current challenges of computing science that are not solved by existing OS: the presence in the network, serviceorientation and, of course, the three major design factors inherited from the evolution of old OS: performance, security and reliability. For all this, our proposal is oriented to increase the level of the abstractions provided by the operating system and their services. This makes possible to offer an OS layer integrated to the network, and security and reliability mechanisms not available in lower levels of the architecture of the OS.

These changes begin by replacing the paradigm that is used. Changing the abstractions that an OS uses is linked to the paradigm used. This new computing paradigm is presented next.

\section{Distributed Goal-Oriented Computing}

In our work we define the concept of Distributed Goal-Oriented Computing as the paradigm where heterogeneous agents can express their desires by using goals. These agents can also fulfill the goals by using automatic composition of services that are available in the cloud. In this section the Distributed GoalOriented Computing paradigm is presented by means of showing the model that defines it and the architecture that gives support to the related model.

\subsection{Goal-Oriented Execution Model}

The Goal-Oriented Execution model is inspired by the classic BDI agent model presented in [17]. In this model there are included the abstraction of agent, knowledge base, services, goals and plans (which are the service compositions) [5]. Its purpose is to define an execution support based on a different computation paradigm that provides the features presented earlier in this document. The execution model operates on an operating system kernel, which provides the other necessary functionality of a common OS, such as memory management, security, etc.

In the Goal-Oriented Execution model, an agent $A$ is defined through the following tuple:

$$
A=\{K B, S S, C P, G S\}
$$

where:

- $K B$ represents the agent Knowledge Base.

- $S S$ represents a Set of Services offered by the agent. This services are used by the agent to perform its goals, but they can also be offered to other agents to help to achieve their own goals. 
- $C P$ represents a set of Compiled Plans provided by the agent to meet its goals.

- $S G$ represents the Set of Goals that the agent wants to achieve.

The services that the agent can offer in the Goal-Oriented Execution model are OWL-S services. An OWL-S service is defined by the tuple:

$$
S_{i}=\{S P, G R, P M\}
$$

where $S P$ is the Service Profile, $G R$ the Grounding and $P M$ the Process Model of the service. The service profile defines what the service does. The grounding defines how to interact with the service and the process model defines how the service is used.

Moreover, OWL-S service process model can be composite processes and atomic processes. A composite process is a set of atomic processes (which have no internal structure and run in a single step) with an internal structure built up by composite and atomic processes and a few control constructs (sequence, if-then-else, choice, etc).

This kind of OWL-S service is a well-defined standard which provides this model enough power to construct all the functionality provided by an agent. The services that make up a plan are the real executable part of a plan. A service $S_{i}$ is also composed of a pre-condition $P$, a post-condition $Q$ and a set of inputs and outputs. The pre-condition $P$ is a prerequisite for the execution of a service. The post-condition $Q$ is the impact that will drive the execution of the service $S$ and it represents the Goal entity that the agent wants to achieve. Both $P$ and $Q$ are defined in the functional aspect of the service profile.

\subsection{Goal-Oriented Execution Architecture}

Since a composition of OWL-S services is a composition of services, which include both atomic and composite processes and control structs, we define a Plan as a process model composed by one or more composite process models (again, including composite services, atomic services and control structs). A Plan defines the way to achieve some results or post-conditions by joining different OWL-S services which can be connected. Composite services or even atomic services can be seen as very simple plans, but we also define a plan as the result of joining different composite services in order to achieve a goal.

To give support to the model presented, a Goal-Oriented Execution architecture has been developed. The architecture is composed by the next components (Figure 1):

- Runtime Engine: The Runtime Engine takes the plans provided by their planners and manages their execution by transferring the service execution to the OS kernel. If necessary, the Runtime Engine invokes distributed services provided by agents that are located in other hosts. 


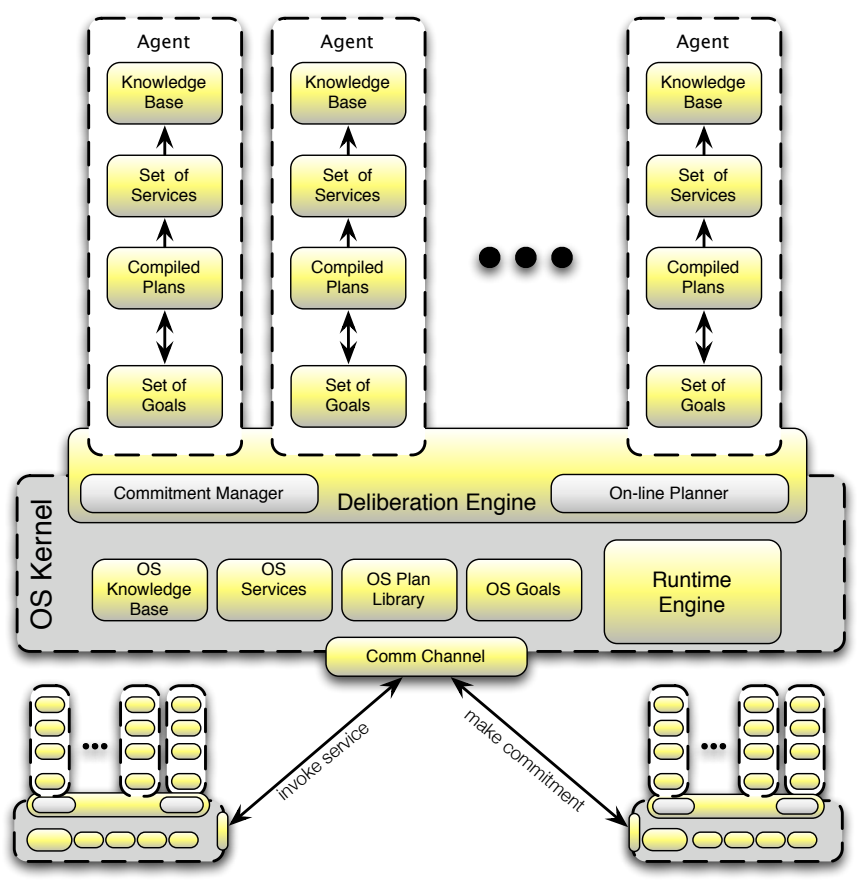

Figure 1: Agent and execution module components

- Deliberation Engine: It is responsible for deciding how and in which order plans are executed. This engine has the ability of negotiating with the service provider agents that are offering the current plan. This engine is permanently running in background evaluating the goals that the agents want to fulfill and selecting them for its completion. This component interacts concurrently with the Runtime Engine, and is composed by the Commitment Manager and the On-line Planner.

- Commitment Manager: Service provider agents negotiate their availability to execute the service with the commitment manager and, if so, executing it within a time frame. To calculate this prediction the agent needs to take into account some points like: (i) the current workload, (ii) the availability of the service at the time of the request and (iii) the availability of the needed hardware and software resources to be able to run. For this work the agent needs the OS assistance. The OS can help the agent to predict if he is going to be able to satisfy the request in the defined temporal bounds, and if so to establish a commitment with the Deliberation Engine. This functionality is offered by the Commitment Manager.

- On-line Planner: it is able to repair or refine running plans. This 


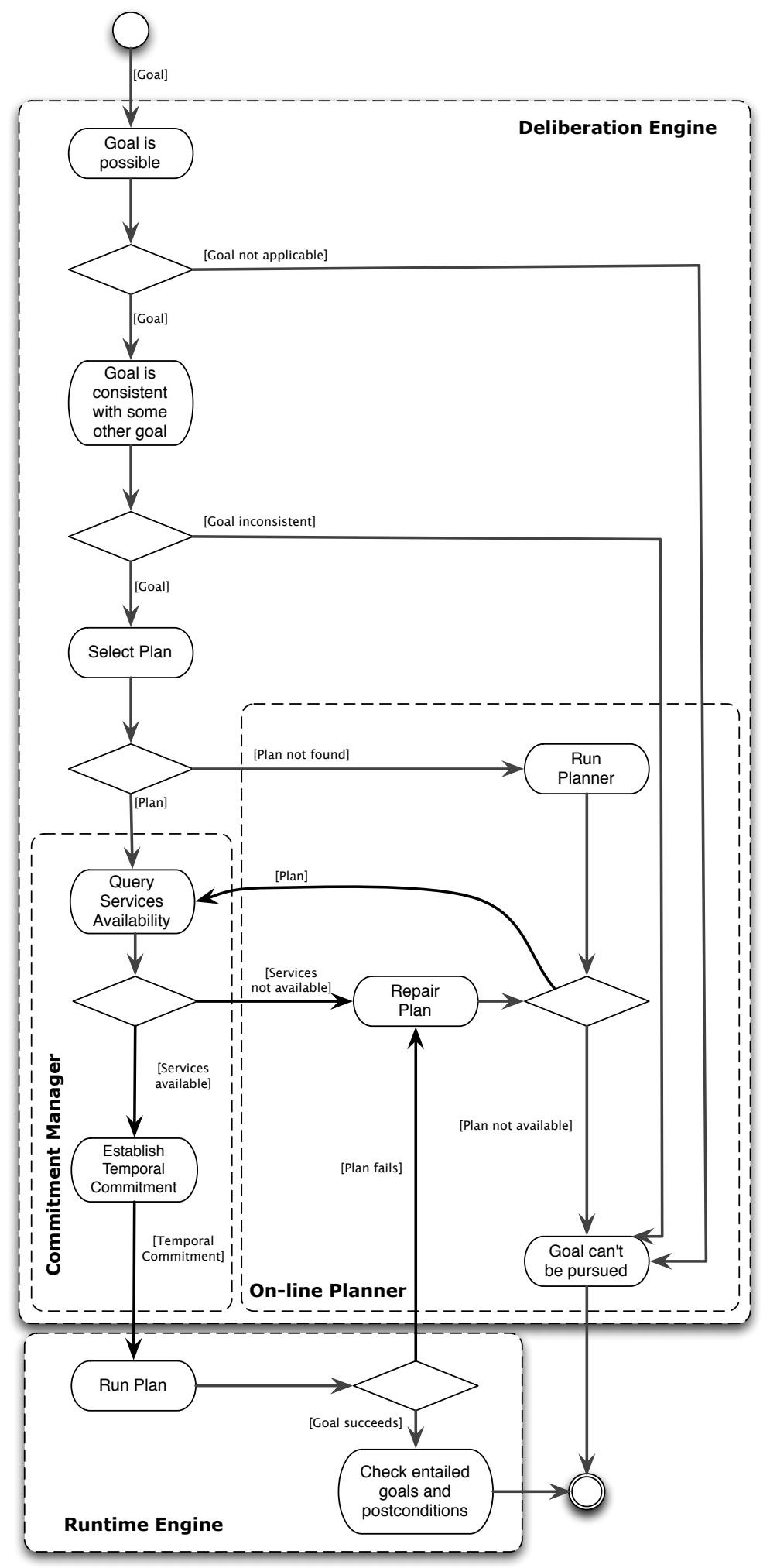

Figure 2: Goal-oriented executive model 
planner is executed concurrently inside the Deliberation Engine and its task is to help the agents to reach a goal when the agent has no precompiled plans that guide him to the goal completion by composing or repairing plans. The On-line Planner uses a TB-CBP (Temporal Bounded Case Based Planner) to generate the plans at runtime. The time required to execute the $\mathrm{TB}-\mathrm{CBP}$ is known and, for this reason, the deliberative process is temporal controlled. Moreover, the TBCBP uses cases that have been executed in the past in order to take a decision about if the calculated plan can be fulfilled in the established temporal bounds.

- OS Goals set: The OS has its own goals to perform the corresponding tasks of an operating system. This set of goals includes all the maintenance tasks and non-critical functionality that the OS must achieve to ensure the proper functioning of the computer.

- OS Knowledge Base: This is the knowledge that the OS has. The operating system uses this knowledge base to perform their goals by means of the services that it can invoke.

- OS Services set: This struct stores the set of basic services provided by the OS. This set of services is used by the OS to provide the basic low-level functionality to the system agents. It includes all the necessary stuff to manage the system and to access to restricted features only available through the OS for security and stability reasons. Some of these features are the communication of system drivers with the hardware, as well as other features that allow the correct interaction among agents, service providers and the operating system.

- OS Plan Library: By means of this library the agent has a set of available pre-compiled plans. This component is created during the OS design phase to provide pre-compiled plans for critical goals that cannot wait for a different composition or cannot vary their execution flow due to security and efficiency reasons.

Under the Distributed Goal-Oriented Computing paradigm the goals that the agents have are sent to the execution module for their achieve. Then, the deliberative engine chooses the appropriate plan to meet each goal. Note that the agent model preserves its desirable features like autonomy and pro-activity since the agent is who activates its own goals when he decides he wants to achieve them. The deliberative engine provides the needed resources to help to achieve the goals. Plans may be provided by the agent itself or can be compounded on-line. These plans are a sequence of services offered by the agents both locally and remotely. It is also an agent choice to share its precompiled plans with other agents. The basic running elements are the services that make the plans. Plans are provided to the module in two different ways: the off-line generation of the plan or the on-line generation of the plan by the On-Line Planner module. Once the plan that meets the active goal is selected, 
the Runtime Engine activates the services that comprise the selected plan. In Figure 2 the deliberation and execution processes are shown.

Once the Goal-Oriented Execution model and architecture have been presented, in next section we are going to show the deliberation process that is used to fulfill the agent's goals.

\section{Deliberation Engine}

The Deliberation Engine is the brain of the execution module. This component is in charge of analyzing the current active goals and helping their achievement. The Deliberation Engine is the root node which manages all the main flow of the execution process. It communicates to the Runtime Engine to run the services, to the Commitment Manager module to manage the temporal commitments of the agents and to the On-line Planner module to compose new plans.

The Deliberation Engine is responsible for deciding what actions should be performed to achieve a goal. This component is in charge of analyzing the current active goals and helping their achievement. The Deliberation Engine is the root node which manages all the main flow of the execution process. To run the services, the Deliberation Engine communicates to the Runtime Engine to indicate the services composition to execute.

Since a goal is activated by an agent until it is achieved, the Deliberation Engine goes through different steps which involve the different components of the execution module. These steps are:

1. Checking if it is possible to activate the goal.

2. Checking if the goal is consistent and there are no conflicts.

3. Asking the On-Line Planner for a set of plans that achieve the goal.

4. Querying the Commitment Manager for a temporal commitment for each service of the plan.

5. If there is no available commitment, asking the On-Line Planner for a new plan or setting the goal as unreachable.

6. Selecting the best plan from the set of plans using the temporal commitments and the historical quality parameter.

7. Sending plan to the Runtime Engine to be executed.

8. If the plan fails, asking the On-Line Planner for a new plan or set the goal as unreachable.

9. When the plan ends, updating the case-base with the results of the commitments.

10. Checking entailed goals and postconditions and setting the goal as reached.

There are two modules that facilitate the deliberation engine to make decisions when it determines the service composition. They are the On-line Planner and the Commitment Manager. 
Table 1: Example of Case-Base of the TB-CBP

\begin{tabular}{|c|c|c|c|c|}
\hline Postcondition & Precondition & Services & Quality & Time \\
\hline B & A & $\{\mathrm{S} 1\}$ & 1 & $4 \mathrm{t}$ \\
\hline C & A & $\{\mathrm{S} 1, \mathrm{~S} 3\}$ & 0.85 & $10 \mathrm{t}$ \\
\hline C & B & $\{\mathrm{S} 3\}$ & 0.85 & $6 \mathrm{t}$ \\
\hline D & $\mathrm{C}$ & $\{\mathrm{S} 6\}$ & 0.9 & $7 \mathrm{t}$ \\
\hline E & $\mathrm{B}$ & $\{\mathrm{S} 7, \mathrm{~S} 10, \mathrm{~S} 11\}$ & 0.76 & $11 \mathrm{t}$ \\
\hline $\mathrm{E}$ & $\mathrm{D}$ & $\{\mathrm{S} 8\}$ & 0.99 & $3 \mathrm{t}$ \\
\hline $\mathrm{E}$ & $\mathrm{D}$ & $\{\mathrm{S} 4, \mathrm{~S} 12\}$ & 0.98 & $7 \mathrm{t}$ \\
\hline F & $\mathrm{C}$ & $\{\mathrm{S} 5, \mathrm{~S} 9\}$ & 0.81 & $7 \mathrm{t}$ \\
\hline $\mathrm{F}$ & $\mathrm{E}$ & $\{\mathrm{S} 13, \mathrm{~S} 14\}$ & 0.98 & $10 \mathrm{t}$ \\
\hline$\ldots$ & $\ldots$ & $\ldots$ & $\ldots$ & $\ldots$ \\
\hline
\end{tabular}

\subsection{On-line Planner}

The responsible entity for providing plans that fulfill the agents' goals in the Deliberation Engine is the On-Line Planner. This planner is built on a CBP (Case-Based Planning) [21]. This CBP has been modified for giving a temporal bounded response in order to have a temporarily predictable execution. This new model, called Temporal Bounded CBP, is composed by the same phases as the classic CBP but these phases have been treated to bound their execution time. Thus, the execution time of the service composition process is known and this time is taken into account when the On-line Planner must build a plan within a maximum time. A general description of the functioning of the $T B-C B P$ on-line planner is shown below.

First, the case structure used in the base-case offered by the TB-CBP is defined as:

$$
<\text { Postcondition, Precondition, }\{\text { Service }\}, \text { Quality, ExecutionTime }>
$$

where:

- Postcondition is the goal wanted to be achieved.

- Precondition are the initial conditions that must be given to start the execution of necessary services to fulfill the goal.

- Service is the list of services that must be executed from the state Precondition to reach the state Postcondition.

- Quality indicates the confidence that the system has about the correct execution of the services.

- ExecutionTime is the time required for the execution of the services. An example of the used case-base is presented in Table 1.

To complete the search of a service composition, the agent will inform about its goal (Postcondition) and its believes (Precondition). With this information, 
the On-line Planner can fulfill a service composition. To do it, the planner extracts cases from the case-base and composes a path between the goal to be achieved until it reaches any of the believes that the agent knows.

Let's imagine the following situation using the information in Table 1. An agent wants to fulfill the goal $F$, and its believes are $\{$ A,B $\}$. The On-line Planner will extract from the case-base all cases that have as Postcondition the goal $F$. For every extracted case the algorithm will come to search in the case-base, but now the Postcondition are the pre-conditions of all the extracted cases (Precondition parameter). This process will follow until it extracts a case whose Precondition is either defined in the agent's believes (Precondition $=A \vee B)$. In Figure 3 we can see the search progress from $F$ to $A$ or $B$. In this case, several plans are possible. In response to the needs of both the agent or the Operating System just one plan will be chosen. If the agent wants to get a result with the best quality then he picks any of the plans marked as (3). It is possible that the agent wants to get a plan that gets the goal as soon as possible. In this case he will choose the plan marked as (1). If the agent wants a plan that meets within a specified temporal bound, i.e. before 22 time units, with the highest quality, in this case he will choose the option (2). As shown, the execution module has the freedom to choose a plan taking into account the agent necessities. This makes the system more adaptable to the agent needs.

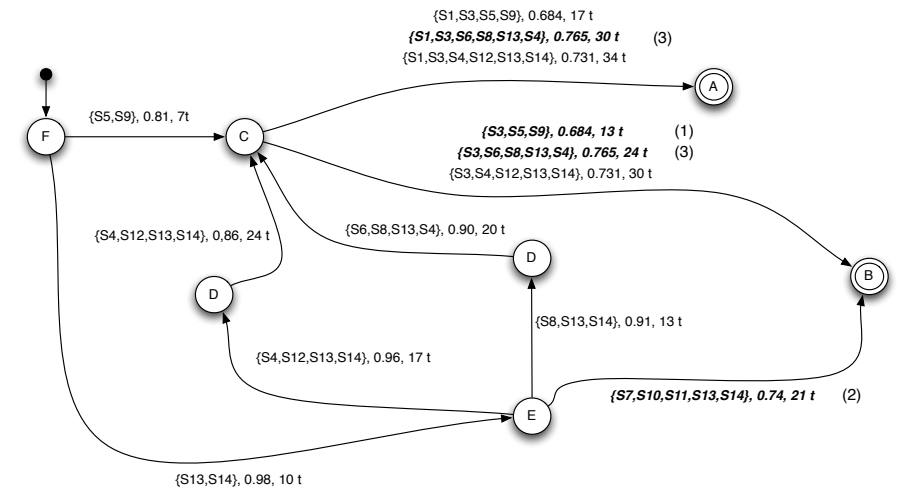

Figure 3: Search sequence in the case-base

Once the plan is calculated by the On-line Planner, the system must verify that all the services associated to the plan are available and how much is the workload of the agents that must execute the services. This function is performed by the Commitment Manager which is seen below.

\subsection{Commitment Manager}

The Commitment Manager is based in a SAES framework [6] which allows us to compose services and to guarantee their correct execution and finalization on time. The main difference with the SAES approach is that by introducing 
the service framework as part of the operating system, it has more information to make better temporal commitments and predictions.

It is possible to identify two main functions in the Commitment Manager. First, it must check if the set of services offered as a plan by the On-Line Planner will be available to fulfill the request and then it must establish a commitment relationship with the agents that provide the selected services (see Figure 4).

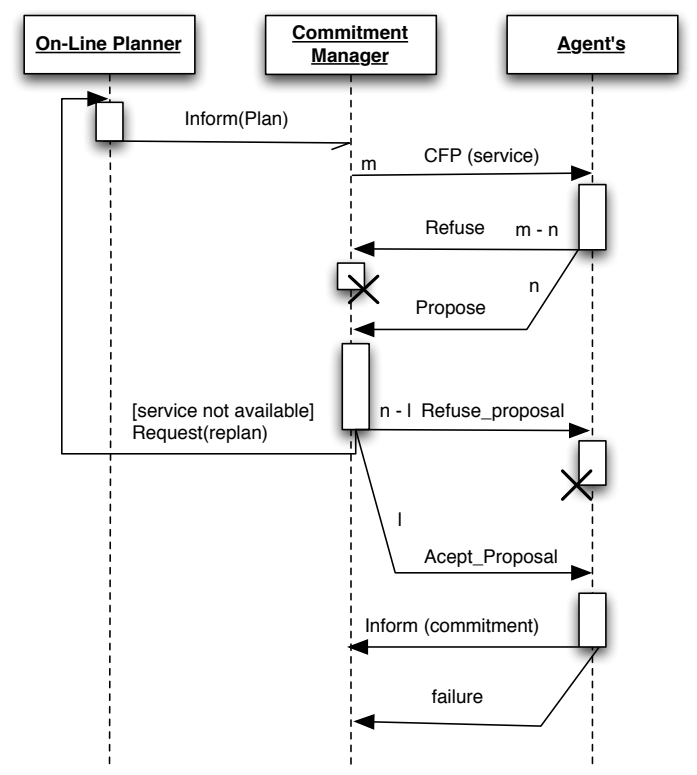

Figure 4: Services Availability Query interaction protocol

To fulfill the first function, the Commitment Manager sends a call for proposals to all agents that can offer the services involved in the service composition. Each agent analyses when he can finish the service, and then each agent returns a proposal to the Commitment Manager. The proposal consists of a tuple:

$$
<T_{\text {start }}, T_{\text {duration }}, P S>
$$

where:

- $T_{\text {start }}$ indicates the moment when the service can start its execution.

- $T_{\text {duration }}$ indicates the necessary time to complete the service.

- $P S$ is the probability of a successful execution.

During the execution of the On-line Planner the system obtains a quality measure which is used to estimate the best plan. In this case, the Commitment Manager calculates a probability that indicates if the agent can complete the service in the time that is indicated taking the workload into account. This 
information is more accurate than the quality obtained in the recovery plan because it takes into account the current situation of the agent that offers the service and the real workload of the system. with all this information, a precommitment between the agent and the Commitment Manager is established.

When all agents have answered to the Commitment Manager, the CM must calculate the success probability associated to the whole service composition. To do that, the Commitment Manager uses the success probability sent to all agents. This success probability is weighted with the information of previous executions of similar services by these agents and that the Commitment Manager has stored. The service composition success probability is calculated as it follows:

$$
P S_{\text {composition }}=\prod_{i=0}^{N} P S_{i} * \omega_{i}
$$

where $\omega_{i} \in[0,1]$ is the weight associated to the service $i$. This weight is related to the previously fulfilled commitments; an agent who has many unfulfilled commitments will have a low weight.

Once the Commitment Manager calculates the service composition success probability, it sends the composed service and its probability $P S_{\text {composition }}$ to the Deliberation Engine. The Deliberation Engine analyses if it is a suitable composition. If it agrees with the service composition, it communicates to the Runtime Engine that the service executions can start. When this is the case, the pre-commitments established with the agents are confirmed by the Commitment Manager. If the Deliberation Engine does not agree with the service composition, the Commitment Manager breaks the pre-commitments, freeing the slack reserved by the agents.

The Commitment Manager is also in charge of ensuring that the acquired commitments are fulfilled. In case that a commitment cannot be fulfilled, the Commitment Manager penalizes the agent which provides the service. This penalty is captured through the weights applied when the Commitment Manager updates the service composition success probability.

\section{Runtime Engine}

The Runtime Engine is the component in charge of managing the entities that are running in the system. This includes driving the execution of the process model of the active plans and scheduling the services that are invoked by a plan, both the local and the remote invocations.

The execution of an atomic service is much like a traditional operating system's process abstraction. These services are scheduled and executed by the Runtime Engine with a proper context. These services have also a life cycle inherited from traditional processes[19]. The states of the service life cycle are: (i) ready to run, (ii) running and (iii) sleeping.

As stated before, the Runtime Engine also manages the life cycle of plans. The execution of plans is made in collaboration with the Deliberation Engine's 


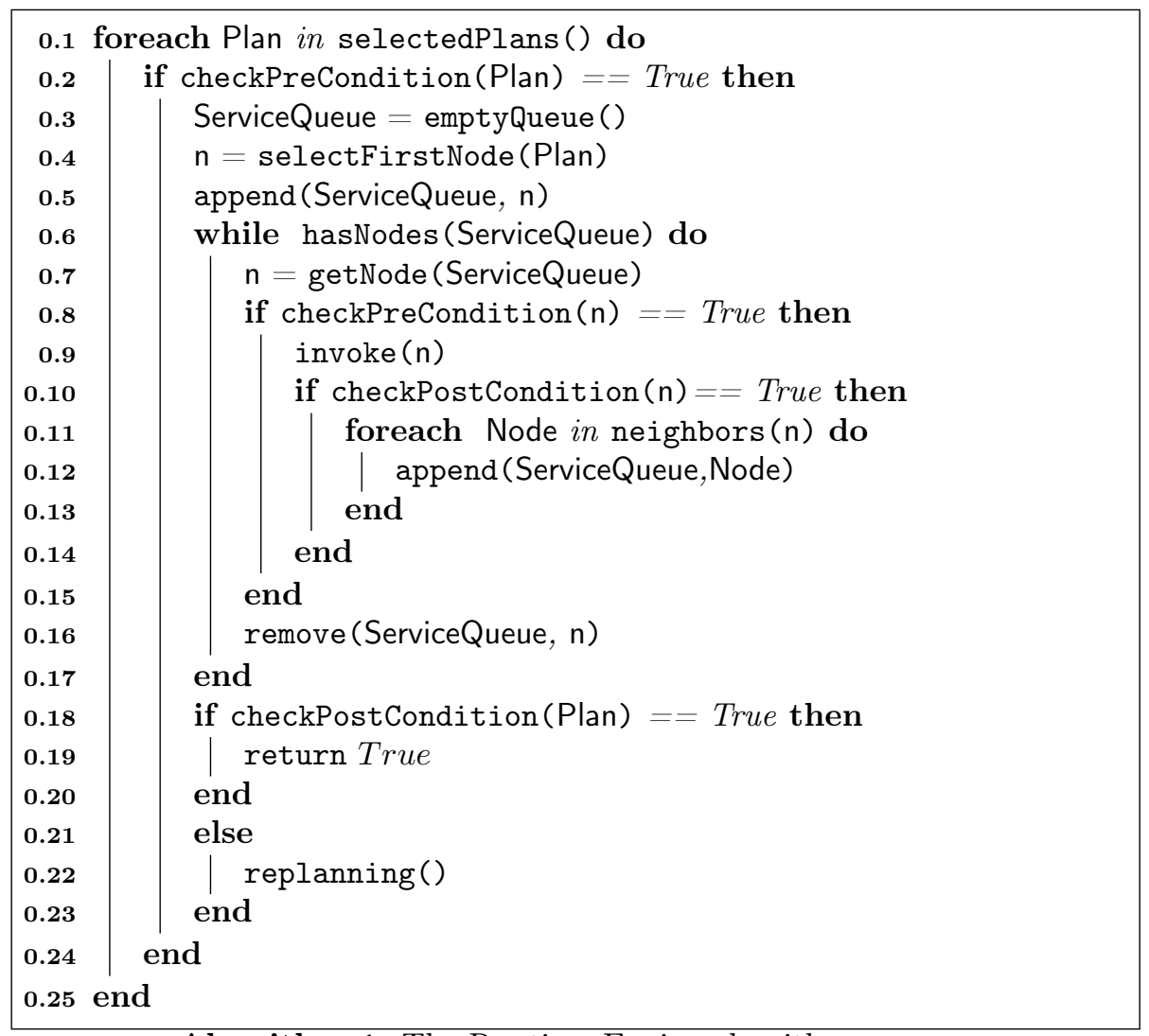

Algorithm 1: The Runtime Engine algorithm

Commitment Manager. While the Commitment Manager is in charge of ensuring that the temporal commitments are achieved, the Runtime Engine checks that every step of the plan is properly executed. This includes to ensure that, before executing a service, all its preconditions are true and that, after executing the service, all their postconditions have been achieved. This part is carried out by following the OWL process model $(P M)$ at each step, following the logical flow that determines its preconditions and postconditions. The task of visiting the process model of each active plan and check the preconditions and postconditions of each node belongs exclusively to the Runtime Engine.

Algorithm 1 shows the steps followed by the Runtime Engine:

1. The Runtime Engine (RE) extracts a plan from the list of selected plans created by the Deliberation Engine.

2. The first action is to check that the plan's precondition is valid and can be executed.

3. At this moment the plan is selected as a running plan. The RE selects the first node of the plan from its service graph and invokes the service by 
appending it to the scheduler's ready queue.

4. Before executing a service the Runtime Engine previously checks its precondition and, after the service execution is finished, it checks the service postcondition. If the postcondition is valid the execution of the plan can continue.

5. Once the service finishes its execution, the RE extracts from the process model all its neighbors and checks their preconditions. These neighbors are all the nodes that are directly accessible from the given node through a control construct.

6. This process continues until the service process model reaches a final node or their services fail and a plan reparation is needed (using the On-line Planner).

7. When the plan finishes, the Runtime Engine checks its postcondition. If it is valid, the goal that has motivated the execution of the plan is marked as pursued. Otherwise, a new plan is requested to the On-line Planner.

The Agent is the main entity that motivates this execution model. Agents can flow through different states, depending on their current role:

- Applicant: The agent has goals to pursue and does not offer any service.

- Provider: The agent offers services to other agents but has no current goal.

- Provider-Applicant: The agent has goals to pursue and also provides some services for both its own use and for other applicant agents use.

- Inert: The agent has neither current goals nor provided services. This is the case when the agent is ready to leave the system.

Once the Runtime Engine executes a plan it notifies the On-Line Planner in order to perform the retain step, this is, to store the new case (whether it is successful or not) to keep the case-base updated.

\subsection{Execution trace}

This section will expose a sample trace where the different steps that this execution module follows to achieve a goal are shown. For simplicity we have prepared a simple scenario with a few elements and a single goal to achieve. To show the flexibility of the system we will simulate an error in the trace, showing the fault tolerance of the module.

In this example there is an agent that acts as an interface of the user (the client agent) and a set of services distributed around the different nodes of the network. Each of these services is provided by an agent and is hosted in a node which is connected to the node where the client agent is hosted. The prepared scenario is designed to perform a very common task: saving a song in an iPod. In this scenario the client agent just expresses its goal (Song in Ipod), and has some previous knowledge in its knowledge base: the audio he wants to save 
(PCM Audio) and some metadata (title, author, genre,...) about the song (Song Metadata). These knowledge items will act as the preconditions of the plan that is going to be executed.

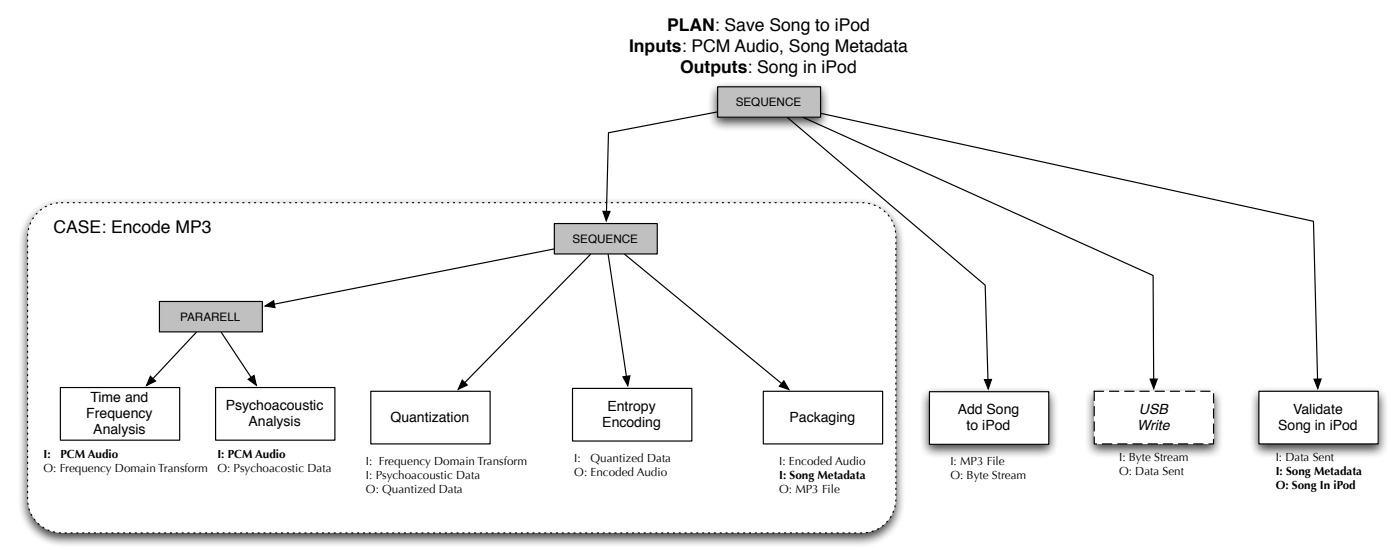

Figure 5: Process model of plan Save Song to iPod

When the client agent activates the goal the Deliberation Engine looks for a plan to fulfill the goal. Since there is not a plan that is able to perform the goal expressed by the client agent, the On-line planner generates a plan that is able to perform the desired goal starting from the known KB items as preconditions. This plan is shown in Figure 5. The iPod only works with MP3 encoded audio (and the precondition expressed by the agent is encoded in raw format), so the generated plan will include the needed services to encode the raw audio to the MP3 format. The services that encode audio were previously executed in the system, that is why there is a Case included in the generated plan in Figure 5. The dashed box represents a service provided by the operating system of the client agent. For this reason the service is hosted in the same node than the client agent.

As an example, we'll follow an execution trace using this plan:

1. Initially, the Deliberation Engine would select a goal of an agent. For simplicity there is only one goal, which is Song in iPod. Since there is only one goal, the Deliberation Engine selects it.

2. The On-line Planner generates a plan to fulfill the goal (Figure 5), as stated before.

3. As long as the plan meets the precondition (the agent knows PCM Audio and Song Metadata), the deliberative engine will select the plan for its execution since its postcondition is compatible with the desired goal (it generates Song in iPod).

4. The first services to be executed are Psychoacoustic Analysis and Time and Frecuency Analysis. Before running them, the Commitment Manager establishes temporal commitments with their hosts. 
5. The Runtime Engine executes the services Time and Frecuency Analysis and Psychoacoustic Analysis, achieving as effects the values Frequency Domain Transform and Psychoacoustic Data. The Commitment Manager checks that the temporal commitments were accomplished, rewards the services and performs the retain stage in the Case-Base of the On-line Planner.

6. Next service is Quantization. After the establishment of the temporal commitments, the Runtime Engine executes the service Quantization, achieving as effect the value Quantized Data. Once again the Commitment Manager rewards the service and retains the case.

7. To show the advantages of running this model, we introduce an error at this point. Let us assume that the service Entropy Encoding is unavailable (the agent that provides the service is not connected, the service is saturated, or maybe the output is not a real MP3 file). This situation generates that the Commitment Manager punishes the case representing the service.

8. At this time, the Runtime Engine would ask the On-line Planner a repair of the running plan to continue the execution of this agent.

9. The planner would return the plan shown in Figure 6. This repaired plan continues where the other plan has failed its execution and replaces the failed service with other structure thanks to other services found in the distributed system. The new plan has a very similar structure but replaces the encoding service with a choice for other three time domain encoding services (PCM Encoding, Differential PCM Encoding and Adaptive PCM Encoding).

10. At this moment the Commitment Manager needs to establish a commitment with the service which ensures a lower execution time and offers a better trust value. To do this, the CM asks the case-base for old trust stored values and asks the providers hosts about their temporal commitments. With this information the Runtime Engine selects for execution the Adaptive PCM Encoding service.

11. Finally the execution of the plan is ongoing through the services Packaging, Add Song to iPod, USB Write and Validate Song in iPod. At each step a temporal commitment is established and the service executed is punished or rewarded depending on the case.

12. When the execution of the service Validate Song in iPod has finished, the client agent has in its knowledge base the fact Song in iPod, so the goal has been achieved and it can be removed from the agent set of goals.

A remarkable aspect of the client agent is that despite the selected plan has failed, it has been able to achieve its goal on a completely transparent way to the agent through the ability of replanning of the execution module. With this module the success degree of goal achievement is higher than on classic BDI systems. This module has also the ability of providing system services for the plan composition, allowing the OS to work with this paradigm, as is the case of the USB Write service. 


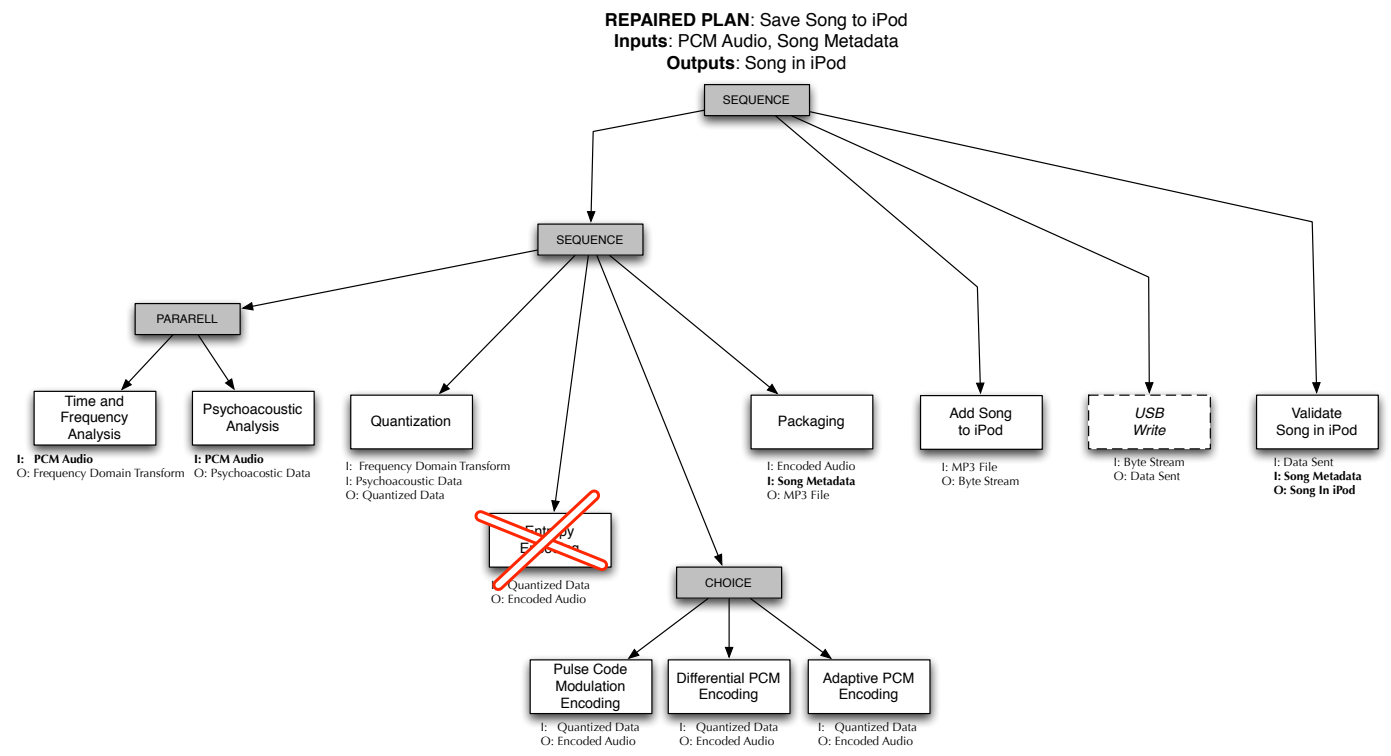

Figure 6: Repaired plan Save Song to iPod

\section{Tests and Results}

In order to evaluate the architecture presented here for the development of goal-oriented operating systems, this work presents a set of tests and results that validate the proposal. A discrete simulator has been developed to test all the features and advantages provided by an Operating System implementing the Distributed Goal-Oriented paradigm. In this section we present how the simulator works and how the different tests that have been done by analyzing what the different components of the proposal (runtime engine and deliberation engine components: commitment manager and on-line planner) contribute to the system.

\subsection{The simulator}

The operating system simulator allows us to test the provided functionality proposed by this work, but avoiding the complexity of developing the full operating system low-level abstractions. This simulator implements the main components of the goal-oriented operating system execution module that are needed for our purposes. This is mainly the execution module, which comprises the runtime engine and the deliberation engine (including the on-line planner and the commitment manager). The runtime engine is in charge of executing the services that are invoked by any running plan. The deliberation engine selects the goals that are activated and finds a plan which performs the goal within a temporal commitment. 
The simulator also supports the representation of a distributed environment, where there are several goal-oriented operating systems which offer their services in a shared network using a common publish-subscribe protocol (like ZeroConf[2] or XMPP[1]). Thanks to this protocol, when an agent registers or unregisters a new service every OS in the same network receives a notification of this event and updates its case-base.

The environment also simulates a global time service which synchronizes the clock in every OS in the network. If a new OS is added to the environment it gets automatically synchronized with the rest of the system. This global time service is very useful for establishing proper temporal commitments and uses known solutions for clock synchronization in distributed real-time systems[12].

Every operating system in the simulation environment has also a communication module that is in charge of managing communications between the nodes of the network. A node is a representation of a goal-oriented operating system. This message passing system simulates a time-bounded environment which allows us for predictability of end to end operations.

Since this is an ad-hoc simulator developed for our OS testing purposes, it allows us to change some parameters in order to explore some interesting behaviors. We can parameterize architectural issues, such as the number of nodes in the network, the number of agents per node, or the number of services or goals that an agent has. The simulator has a scripting system that loads a configuration for the desired environment. The script can define the initial configuration of the environment (number of nodes, agents, distribution of the services by agent, goals, preconditions, etc), setting up the scenario that is desired for the simulation. It can also schedule different events that will be processed during the simulation in order to change the environment at runtime.

To compare the different behaviors, a set of internal parameters can be changed. The probability that a service fails during its execution is parameterizable in the simulator. This way we can check how the operating system behaves in a fault tolerant environment. We can also modify the precision of calculating a temporal commitment in the simulator. Changing the prediction algorithm or the quality of the algorithm itself we can compare different nodes having responses that are not equal for a same request. This is a good way of detecting how the system adapts itself to a changing environment. This kind of tests will be presented in next sections.

Below all the tests have been conducted using the same methodology and with at least 20 repetitions to extract a statistically significant mean and standard deviation. This test of significance ensures with great confidence that the null hypothesis was avoided.

Next, the set of tests performed in this work are presented. They have been divided into two main test suites: Deliberation Engine Tests, where its main components have been tested (Commitment Manager and On-line Planner), and Performance Tests, where some advantages of this distributed system are presented. 


\subsection{Deliberation engine Tests}

The deliberation engine is the component that introduces a reasoning process in the proposed operating system. It is in charge of selecting the best available services that can fulfill the activated goals and with the best possible conditions. The deliberation engine components that perform this functionality are the Online Planner and the Commitment Manager. In this work we have developed a set of tests in order to validate their expected functionality.

\subsubsection{Commitment Manager}

The main aim of the Commitment Manager (CM) is to establish temporal commitments between a service provider and a client. When the client invokes a service he needs to communicate to the service's Commitment Manager to get a proper time prediction of when the service response is going to be ready. This prediction is not an easy estimation, since there are lots of factors than can influence in the results (mainly, the workload of the system). The CM must work side by side with the Runtime Engine, which schedules all the running services in the resource (the microprocessor). The scheduling algorithm is very important for the prediction task, since it has to be able to accomplish the established temporal commitments. At the same time, it should get a good performance and a high degree of interactivity in the system.

The way to do this is through resource reservation. The Runtime Engine reserves at least the $50 \%$ of the current remaining processor using first-come priorities. This scheduling algorithm ensures that each service will have a minimum of resources allocated for its execution. This means that if a service has a $50 \%$ of processor and another service has a $25 \%$ of processor, since the first service has twice the allocated resources, it will work twice faster. This is a pessimistic case because the slack time is shared by all the running services.

This algorithm ensures that a running service has a percentage of processor assigned, so it is easy for the Commitment Manager to calculate the response time and establish a temporal commitment with the client. Since the priority is assigned using first-come preferences, and it always assigns the half of the remaining processor time, the Commitment Manager can calculate the response time $(\Re)$ using the equation showed in 3 .

$$
\Re_{P}=2^{P} * W C E T_{P}
$$

Where, $P$ is the priority of the service and $W C E T_{P}$ is the worst-case execution time of the service, which is provided by the service provider agent. This is a pessimistic approach since it ignores the slack time that is gained when the processor is idle. Improvements to this algorithm are being prepared and will be proposed in future work, including priority promotions when a service finishes and estimation of time gained when there are priority promotions.

Below are the tests that check the proper functionality of the Commitment Manager. These tests show how the system tries to select always the best services that are available to perform the agents goals. 


\section{Test 1: Trust evolution for different deadline predictions}

In this experiment we are going to show how the trust that a client node has in different provider nodes evolves as time passes, focusing their requests on the more reliable nodes. The trust will be changing due that not all the nodes in the distributed system have the same accuracy when calculating the deadline predictions.

The first experiment has being designed using the following scenario at the initial state:

- There are 3 agents registered in the system: 1 client agent and 2 provider agents, which offer the same service with the same precondition $\mathrm{P}$ and postcondition Q.

- The network is composed by 3 nodes: Each agent is hosted in one of the three nodes in the same network.

- The client agent has the necessary knowledge to run the service $(\mathrm{P})$ and activates the goal $\mathrm{G}$ which is the same as the two services postconditions (this is, $\mathrm{G}=\mathrm{Q}$ ).

- The nodes that host the service have different accuracies to calculate the response time:

- First node has an accuracy of $90 \%$ calculating the deadline prediction of the response time (called GoodProviderHost).

- Second node has an accuracy of $20 \%$ calculating the deadline prediction of the response time (called BadProviderHost).

Each experiment makes a request to any of the available services every time step. This is, the client agent activates its goal and selects a plan to perform its goal. After the execution of the service, the agent resets its knowledge base and re-activates the goal once more. As time passes, the case-base acquires more experience about the nodes confidence. Figure 7 shows the results of running this test. The $\mathrm{X}$ axis represents time (in simulator steps) and the $\mathrm{Y}$ axis represents the cumulative sum of services provided by each node, which is a good representation of the trust that the client has in each node. At the start time, the trust in each node is equal. This is because there are no previous known experiences and the case-base of the client is empty, so the client has the same trust on each provider. As time passes, the number of invocations to each node varies due to the accuracy of the BadProviderHost is not very good and he fails continuously when calculating a proper response time. This makes his trust value going down and, therefore, most of the invocations are done to the GoodProviderHost, as is presented in the related figure.

Note that the Deliberation Engine is not only using the trust value (extracted from the case-base) to determine which service provider to choose. The Deliberation Engine gives a chance to other providers by using an on-line learning algorithm[13] which decides to explore or exploit its solutions. This is done by 


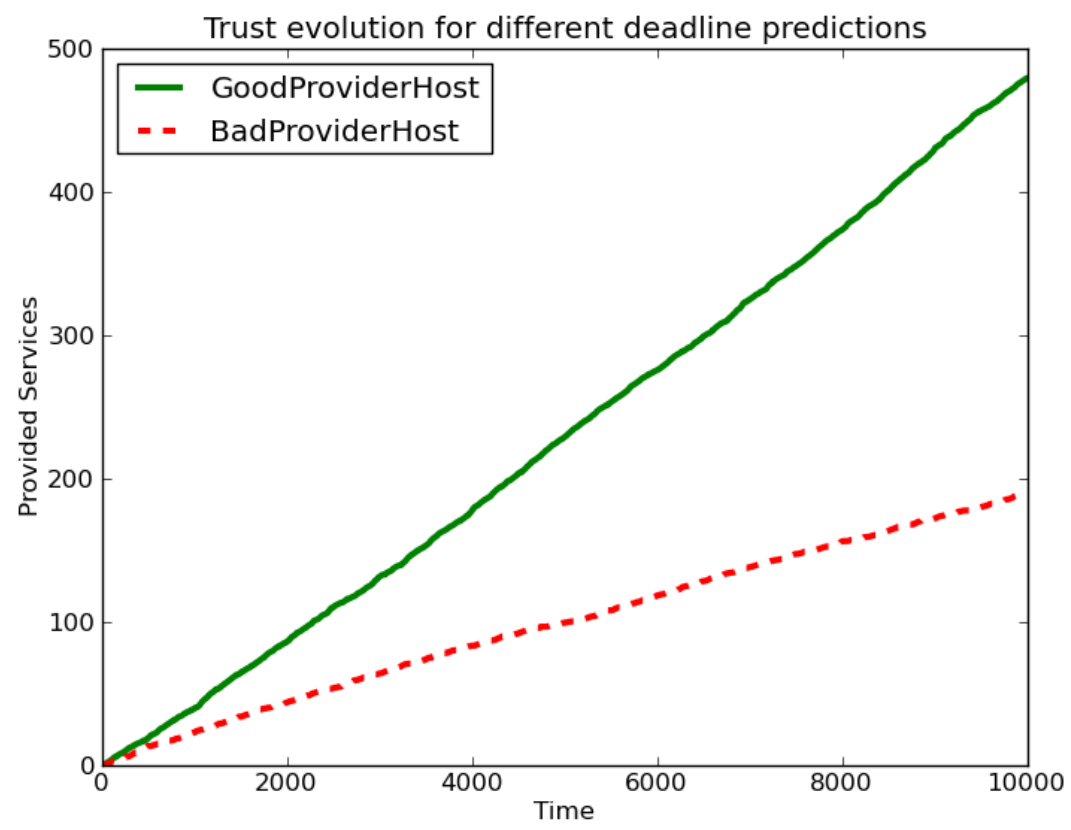

Figure 7: Test 1: Trust evolution for different deadline predictions

adjusting a threshold value during the execution of the operating system. That is why the BadProviderHost provided services are not stuck. They grow more slowly than the GoodProviderHost ones, but sometimes have a new chance.

\section{Test 2: Trust evolution in a bigger scenario}

This experiment shows a similar approach to the previous study (Test 1). The main difference of this test is the size of the agents and nodes sets, which is bigger than in Test 1 . This test shows how the trust in the nodes with a good deadline accuracy grows while the system learns about the environment. The scenario is designed with the following elements:

- There are 51 agents registered: 1 client agent and 50 provider agents which provide the same service.

- There are 51 nodes in the network: Each agent is distributed in one node. Only one agent per node.

- The client activates the goal that invokes the service offered by the providers.

- The deadline accuracy of the nodes is distributed equally in four groups: $5 \%, 33 \%, 67 \%$ and $100 \%$. 


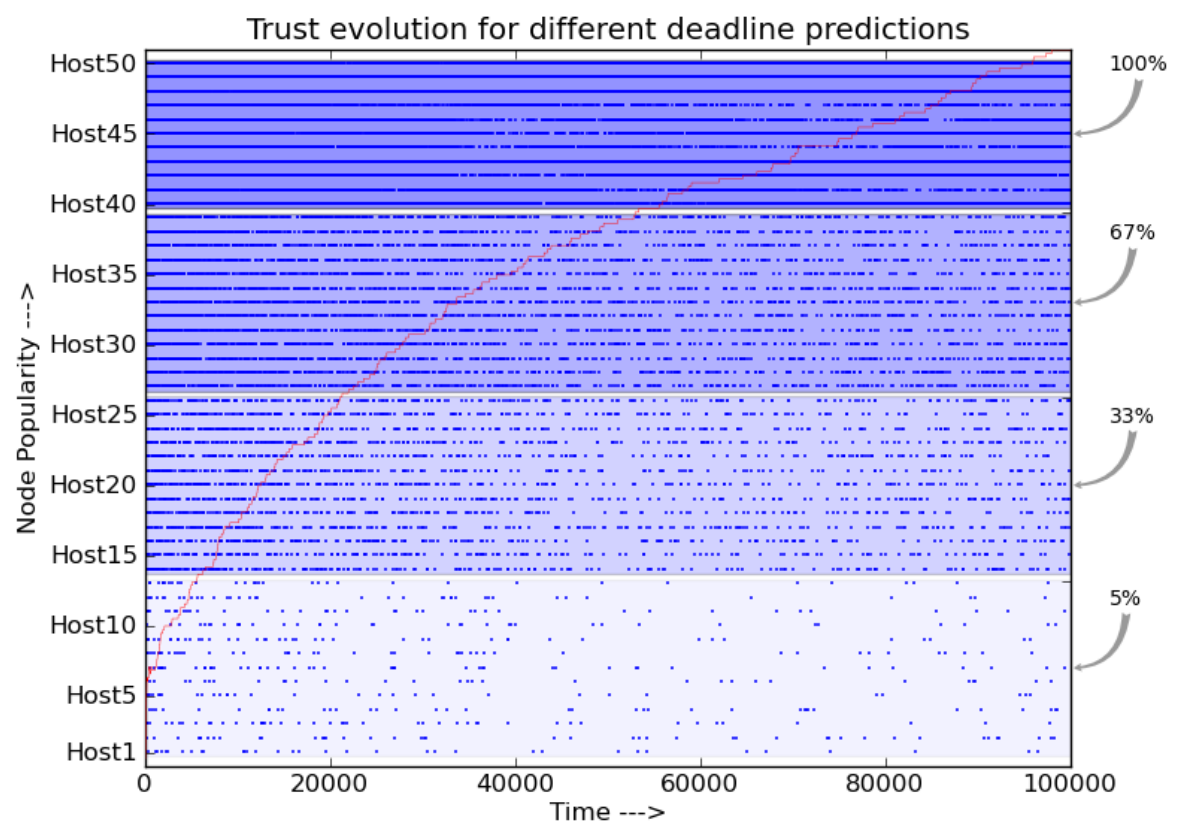

Figure 8: Test 2: Trust evolution in a bigger scenario

The execution of this experiment is equal to the execution of Test 1 . The client agent resets its knowledge base and re-activates the goal every time step. Figure 8 shows the results of the experiment. The $\mathrm{X}$ axis represents once more the time. The $\mathrm{Y}$ axis represents if a service was requested by the client at each time instant. Each dot represents a request to the node that is represented in the Y axis. So, the density of the dot cloud shows how popular is a group of nodes. When the density is large enough and the dots are very close, the representation becomes a straight line.

The middle line is just a mark to divide the dot cloud in the dense area (top-left) and the sparse area (bottom-right). Both areas show how the vast majority of the service requests are in the dense zone. This is again because the OS case-base learns, as time passes, which hosts are more reliable. These results demonstrate that the behavior of the system is what was expected. As we increase the time, the number of requests to the less confident nodes gets decreased.

\section{Test 3: Adaptive Operating System}

This experiment shows how the Operating System is able to adapt itself to changes in the environment. The adaptation of the system is very important, since it allows the system to have a dynamic behavior which is able to re- 
configure itself to take full advantage of current circumstances. For this test we have designed an scenario formed by the following elements:

- There are 5 agents registered: one client agent and four provider agents, offering the same service.

- There are 5 nodes in the network: each agent is distributed in a different node.

- The client activates the goal that invokes the service offered by the providers.

- The accuracy of the nodes at the initial step is distributed as follows:

- Host1: $100 \%$

- Host2: $75 \%$

- Host3: $50 \%$

- Host4: $25 \%$

In this experiment we are going to change the accuracy of some of the nodes to show how the system adapts itself on changing environments. We are going to activate 3 events to change the environment. Specifically, the following events have been scheduled:

- Step 50000: Host 1 accuracy decreases to $20 \%$

- Step 300000: Host 3 accuracy increases to $80 \%$

- Step 600000: Host 4 increases to $90 \%$ and Host 2 decreases to $20 \%$

Figure 9 shows how the trust of the nodes ( $\mathrm{Y}$ axis) changes when the environment undergoes these major changes (marked with the vertical bounding boxes). This trust value represents the trust that the client node has in the other nodes. Adaptation takes time to occur due to the learning algorithm that the deliberation engine is applying. In step 50000 we can see how the Host 1 stops increasing its trust due to the first event. Note that this change takes some time to occur. When the second event occurs (step 300000), the trust value of Host 3 begins to increase (its deadline prediction is improved by $80 \%$ ). Meanwhile the Host 1 trust continues decreasing and the other two hosts maintain their trust value. This third event changes again the system behavior, giving more trust to the Host 4, which has increased its accuracy to $90 \%$. Its trust is growing quickly since its new accuracy is quite good. Parallel to this, Host 2 begins decreasing its trust value.

These results show how the operating system adapts itself when unexpected events change the known environment. In this experiment the client agent changes its trust in the different nodes of the network, changing consequently the number of requests done to each one of the nodes. 


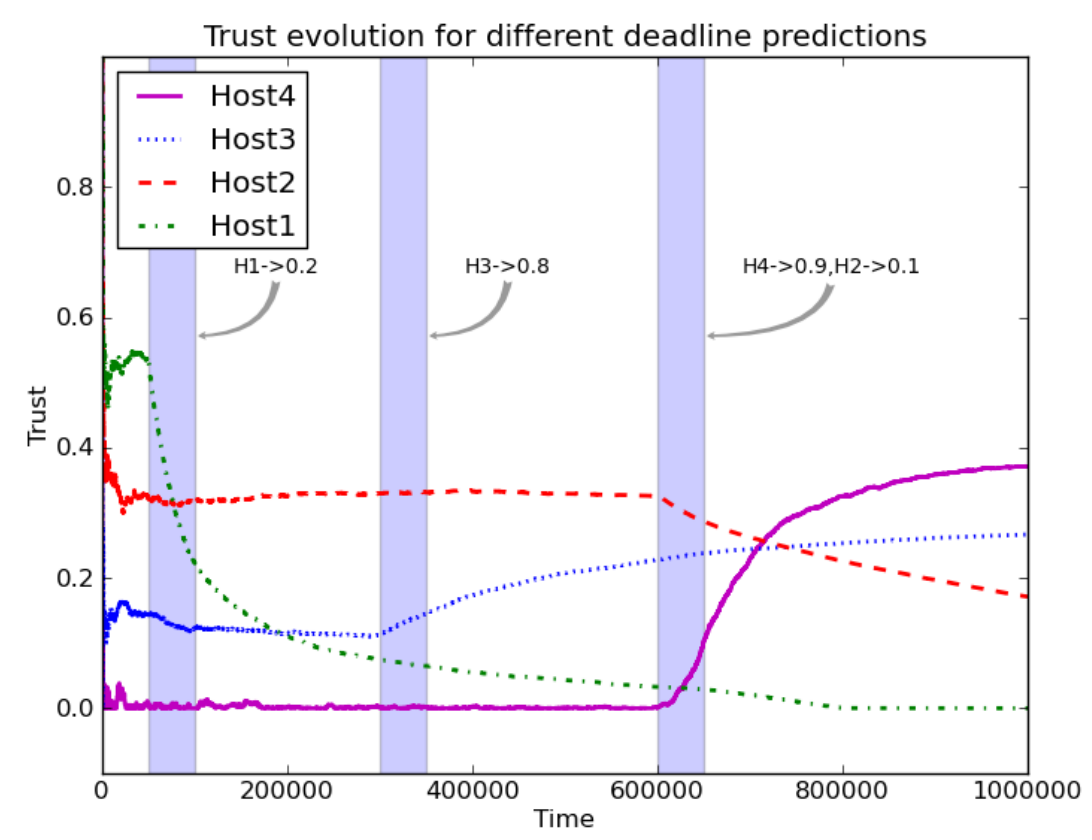

Figure 9: Test 3: Adaptive Operating System

\subsubsection{On-line Planner}

The On-line Planner is the component that allows to compose plans that fulfill the agents goals. This planner uses a time-bounded case based planner (TB-CBP) to create the requested plans by reasoning about past cases. Using a planner to achieve the active goals provides agents an interesting feature: plan repairing, which makes the operating system fault tolerant. This is the functionality that we are going to check with these tests. How the system increases its fault tolerance in unreliable environments and how this affects to the rate of completed goals.

\section{Test 4: Fault-tolerant operating system}

This test has as objective to check how the operating system is able to complete the goals that are active, even if a service execution fails and the plan becomes unuseful. In order to do that, the simulator can be parametrized with an error probability, which defines the probability of a service to fail. This test is defined with the following elements:

- Only 1 host is created, there is no need of distributing the test in this case.

- There are 50 registered agents, each of which has 50 goals to activate. 
- There are 300 services equally distributed throughout all agents.

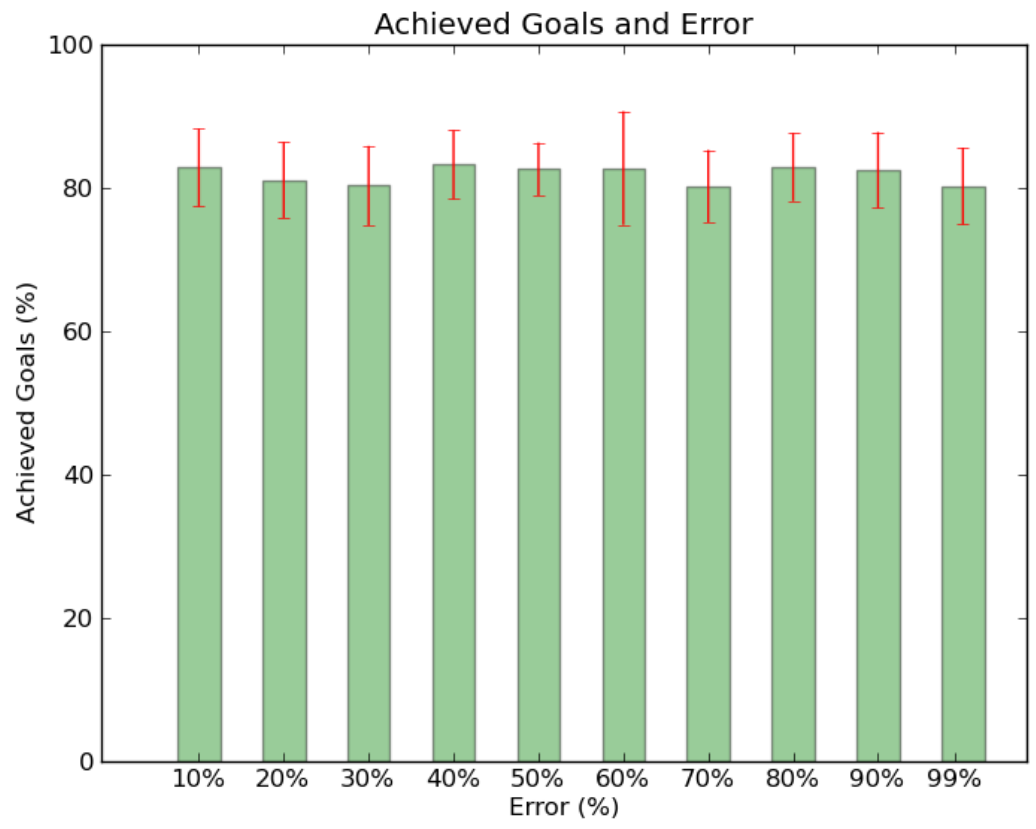

Figure 10: Test 4: Fault-tolerant operating system

In this experiment all the goals, services and agents knowledge items are randomly generated. There is only one parameter that will be changed during the test, the error probability. This parameter will be changed from $10 \%$ to $99 \%$ in steps of 10. Figure 10 shows the results of this experiment. The $\mathrm{X}$ axis shows the error probability assigned to the services. The $\mathrm{Y}$ axis shows the percentage of success for all the goals activated. Note that the percentage of success is not $100 \%$, since the data is randomly generated and there is not always a path from the preconditions to the goals. What is shown in Figure 10 is that the percentage of success of the goals is constant, despite the error probability that the services have. These results are so relevant because they conclude that the proposed operating system is highly fault-tolerant.

\section{Test 5: Trust evolution and multiple errors}

This experiment shows how the combination of previous experiments can affect to the trust of the nodes of the system. This experiment combines the error probability of the running services and the accuracy of the response time calculated by the Commitment Manager.

This experiment has the following scenario: 
- There are 5 agents registered in the system: 1 client agent and 4 provider agents.

- The network is composed by 5 nodes: Each agent is hosted in one of the five nodes.

- The client agent has the necessary knowledge to run the service (P) and activates the goal $\mathrm{G}$ which is the same that the two services postconditions $(\mathrm{G}=\mathrm{Q})$.

- All services have the same behavior but the nodes that host the service have different accuracies to calculate the response time and different error probabilities for the services:

- Host1 has an accuracy of $90 \%$ calculating the deadline prediction and a service error probability of $10 \%$.

- Host2 has an accuracy of $90 \%$ calculating the deadline prediction and a service error probability of $90 \%$.

- Host3 has an accuracy of $10 \%$ calculating the deadline prediction and a service error probability of $10 \%$.

- Host4 has an accuracy of $10 \%$ calculating the deadline prediction and a service error probability of $90 \%$.

Figure 11 shows the results of this test. These results show that there is no relevant difference between nodes with different configurations. The client does not discriminate on the basis of the situation that generated an error (a bad deadline prediction or a service error). What the client can see is that the service has not been provided conveniently (maybe its execution failed or was not provided in time), so the provider is punished. The figure shows how Host 1 , which is the most reliable overall, has the higher number of requests. On the other hand, Host 4 is probabilistically the less reliable node, thus it has the lower number of requests.

\subsection{Test 6: Distributed Computing Performance Tests}

Finally, a performance test has been done to check how this computing paradigm can improve the execution of goals. The Operating System implementing the Distributed Goal-Oriented Computing paradigm has a great impact in the performance of the system. Having an Operating System that not only helps agents to perform their goals, but also searches services to compose the plans on other hosts, largely increases the concurrence of the distributed system.

Test 6 (Figure 12) shows how increasing the number of nodes that offer services ( $\mathrm{X}$ axis) decreases the mean time for achieving goals ( $\mathrm{Y}$ axis). This behavior is highly significant as nodes are added to the network. To run this test, a large enough set of goals has been activated at every experiment. Each 


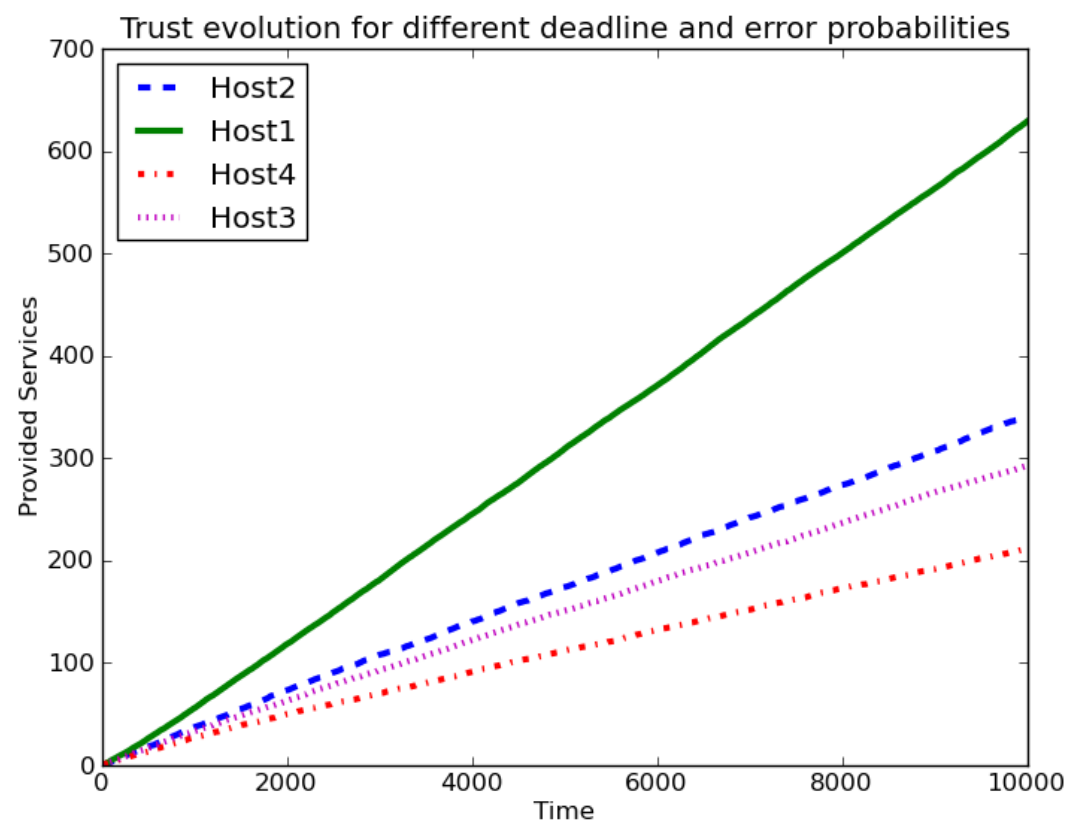

Figure 11: Test 5: Trust evolution and multiple errors

experiment has a different number of nodes (1 to 50) and the agents are distributed equally around the nodes. This way we can perform the activated goals with a higher degree of concurrency and, accordingly, with less time.

We can see in this experiment how important is to increase the amount of nodes in the network. Specifically, the first ten nodes contribute with a great impact to decrease the time needed to fulfill the activated goals. Experiments with 10 or more nodes do not have as much impact as the first experiments, but are always decreasing. In conclusion, the ability of distributing the execution in an automatic and transparent way increases reasonably the performance of the system.

\section{Conclusions}

We have presented in this work a Distributed Goal-Oriented Computing paradigm based on the automatic composition of plans. These plans are formed by distributed services provided by agents. Agents are also the entities who express their own goals and try to fulfill them by means of the plans. To implement this paradigm an execution module for a Goal-oriented Operating System has been designed. The OS purpose is to help agents to achieve their goals by means of a service-oriented approach. 


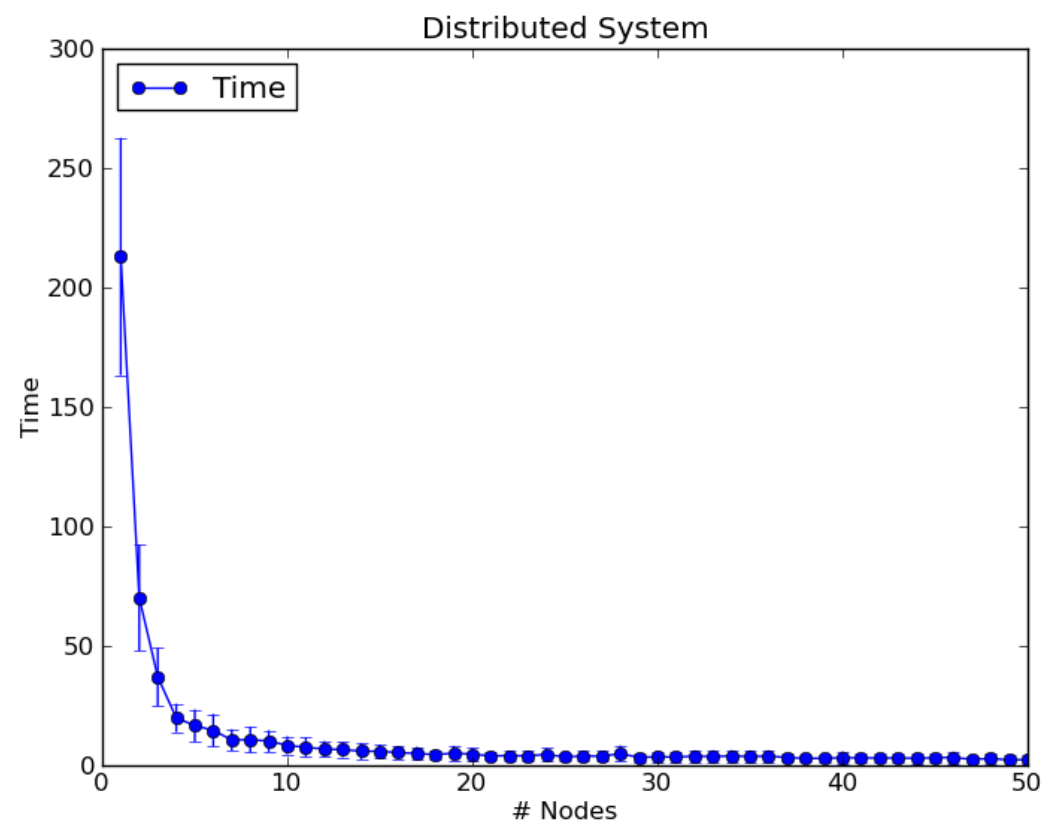

Figure 12: Test 6: Distributed Computing

The OS execution module is divided in two components which are in charge of performing this paradigm. The Deliberation Engine obtains the services needed to achieve the agents goals and stores them in a case base to reason about past cases. This component also takes time and trust constraints into account. This is done either to obtain a result before a deadline, or just to improve the quality of the result.

The case base introduced in the Deliberation Engine uses a Temporal Bounded CBP algorithm to obtain plans that guarantee their execution before a deadline (using the temporal commitments given by the Commitment Manager) and that have a high success degree (reasoning about the trust stored in the case base). This TB-CBP has allowed us to compose on-line plans that give solutions to the goals of the agents following temporal constraints. To guarantee that the agents execute their services before their deadline, the Deliberation Engine provides a Commitment Manager which is in charge of analyzing the workload and establishing a temporal commitment between the agents and the Deliberation Engine.

The results of this work have shown how the Operating System adapts itself to the environment where it is deployed. It selects the providers which offer better temporal commitments and trust values and distributes the workload around these providers proportionally. Also, having an On-line Planner in the 
OS makes it more reliable and fault-tolerant. This is because, even if a service execution fails, the OS will look for a new plan transparently and without user interaction. In fact, the user is not even aware of this.

This proposal opens the possibility of designing service-based operating systems directed by goals using this paradigm. These OS can be extended continuously with new services and plans driven by the user needs. These plans are added by means of the services offered by other users and by their composition, thanks to the new goals defined by the users. The OS architecture defined in this work allows us to use this computing paradigm, since there are some capabilities that only the OS can provide (like soft real-time constraints and temporal commitments).

\section{Acknowledgments}

This work is supported by TIN2008-04446 and TIN2009-13839-C03-01 projects of the Spanish government, PROMETEO/2008/051 project, FEDER funds and CONSOLIDER-INGENIO 2010 under grant CSD2007-00022.

\section{References}

[1] XMPP Pub-Sub: http://www.xmpp.org/extensions/xep-0060.html.

[2] Zero Configuration Networking: http://www.zeroconf.org.

[3] J. S. Breese, D. Heckerman, and C. Kadie. Empirical analysis of predictive algorithms for collaborative filtering. In In Proceedings of the 14th Annual Conference on Uncertainty in Artificial Intelligence (UAI98), pages 43-52. Morgan Kaufmann, 1998.

[4] T. Cortes, C. Franke, Y. Jégou, and T. Kielmann. XtreemOS: a Vision for a Grid Operating System. White paper, Jan 2008.

[5] L. de Silva and L. Padgham. Planning as needed in BDI systems. International Conference on Automated Planning and Scheduling, 2005.

[6] E. Del Val, M. Navarro, V. Julian, and M. Rebollo. Ensuring time in service composition. In 2009 IEEE Congress on Services (SERVICES 2009), volume 1, pages 376-383. IEEE Computer Society, 2009.

[7] D. Greenwood and M. Calisti. An automatic, bi-directional service integration gateway. In Proc. Workshop on Web Services and Agent-Based Engineering (WSABE'2004), 2004.

[8] J. N. Herder, H. Bos, B. Gras, P. Homburg, and A. S. Tanenbaum. MINIX 3: A Highly Reliable, Self-Repairing Operating System. Operating System Review, Jan 2006.

[9] G. C. Hunt, J. R. Larus, M. Abadi, M. Aiken, P. Barham, and et al. An Overview of the Singularity Project. MSR-TR-2005-135, Jan 2005. 
[10] G. C. Hunt, J. R. Larus, D. Tarditi, and T. Wobber. Broad New OS Research: Challenges and Opportunities. Proceedings of the 10th Workshop on Hot Topics in Operating Systems, Jan 2005.

[11] I. Johnson, B. Matthews, and C. Morin. XtreemOS: Towards a Grid Operating System with Virtual Organisation Support. UK eScience All Hands Meeting, Jan 2007.

[12] H. Kopetz and W. Ochsenreiter. Clock Synchronization in Distributed Real-Time Systems. IEEE Transactions on Computers, C-36(8):933 -940, aug. 1987.

[13] R. Michalski, J. Carbonell, and T. Mitchell. Machine learning: An artificial intelligence approach, volume 1. Morgan Kaufmann, 1985.

[14] A. Montz, D. Mosberger, S. O'Malley, L. Peterson, and et al. Scout: A communications-oriented operating system. Hot OS, Jan 1995.

[15] J. Palanca, V. Julian, and A. García-Fornes. A goal-oriented execution module based on agents. In 44th Hawaiian International Conference on System Sciences, page 277, 2011.

[16] R. Pike, D. Presotto, K. Thompson, and H. Trickey. Plan 9 from Bell Labs. Computing Systems, 8(3):221, Jan 1995.

[17] A. Rao and M. Georgeff. BDI agents: From theory to practice. Proceedings of the first international conference on multi-agent systems (ICMAS95), pages 312-319, Jan 1995.

[18] R. Rashid, D. Julin, D. Orr, R. Sanzi, R. Baron, A. Forin, D. Golub, and M. Jones. Mach: a system software kernel. COMPCON Spring '89. Thirty-Fourth IEEE Computer Society International Conference: Intellectual Leverage, Digest of Papers, pages 176-178, 1989.

[19] D. Ritchie and K. Thompson. The UNIX time-sharing system. Communications of the ACM, Jan 1973.

[20] E. Sirin and B. Parsia. Planning for semantic web services. In Proc. Workshop on Semantic Web Services: Preparing to Meet the World of Business Applications, 2004.

[21] L. Spalzzi. A survey on case-based planning. Artif. Intell. Rev., 16(1):3-36, 2001.

[22] M. Wooldridge and I. Dickinson. Agents are not (just) web services: considering BDI agents and web services. Proc. of SOCABE'2005, Jan 2005. 